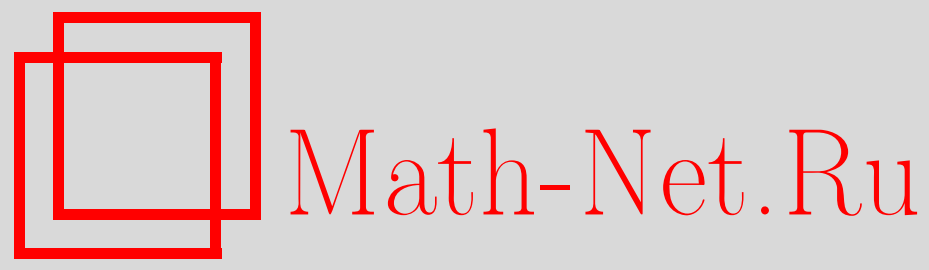

А. М. Вершик, С. А. Евдокимов, И. Н. Пономаренко, Алгебры в планшерелевой двойственности и алгебраическая комбинаторика, Функи. анализ и его прил., 1997, том 31, выпуск 4, 34-46

DOI: https://doi.org/10.4213/faa490

Использование Общероссийского математического портала MathNet.Ru подразумевает, что вы прочитали и согласны с пользовательским соглашением http://www . mathnet.ru/rus/agreement

Параметры загрузки:

IP : 54.92 .164 .108

26 апреля 2023 г., 13:08:25

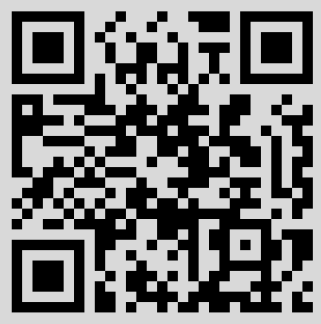


Функииональньй анализ и еәо приложения

1997, т. 31, вып. 4, с. 34-46

УДК 517.9

\title{
Алгебры в планшерелевой двойственности и алгебраическая комбинаторика *
}

\author{
(c) 1997. А. М. ВеРшик, С. А. Евдокимов, И. Н. ПономаРенко
}

В. И. Арнольду кего шестидесятилетию

\section{$\S 1$. Введение}

В настоящей работе устанавливается связь между $C$-алгебрами, являющимися одним из основных понятий алгебраической комбинаторики, и важным классом биалгебр, а именно, алгебрами в планшерелевой двойственности. Мы также указываем новую аксиоматику последнего класса.

Ассоциативные схемы, изучаемые в алгебраической комбинаторике, приводят к матричным алгебрам с выделенным линейным базисом, обладающим особыми свойствами. Этот базис в действительности определяет коммутативное умножение (точнее говоря, коумножение) и потому биалгебру, представляющую собой векторное пространство с согласованными между собой умножением и коумножением. Естественно возникающая при этом задача состоит в описании получающейся структуры в абстрактных алгебраических терминах. Преимущество подобного описания состоит в возможности построения (и последующего применения) общей теории представлений. Получающаяся таким образом категория может включать объекты, не имеюшие упомянутых выше матричных представлений, и тогда проблема существования комбинаторных объектов (таких, как ассоциативные схемы или блок-схемы) трансформируется в проблему поиска специальных представлений подходящих алгебраических систем.

Попытки таких конструкций привели к понятию $C$-алгебры (см. [1] и $\S 3$ настоящей работы). Однако, как оказалось, это понятие не вполне адекватно описывает ситуацию, возникающую в алгебраической комбинаторике: аксиомы $C$-алгебры не включают естественного требования положительности, которому удовлетворяют все «комбинаторные» алгебры. Следует отметить, что, в то время как для коммутативных $C$-алгебр соответствующее дополнительное условие (положительность структурных констант и констант Крейна) легко выписывается (см. [1]), в общем случае ситуация оказывается более сложной.

В 1972 г. один из авторов (см. [7]), обобщая теорию двойственности ТанакиКрейна, Хопфа и др., предложил новое, более слабое, чем в алгебрах Хопфа, согласование двух умножений в двойственных пространствах или, на другом языке, умножения и коумножения, введя понятие алгебр в положительной двойственности. С. В. Керов в [5] изучил это понятие и, в частности, его конечномерный случай (который в основном и нужен для комбинаторики). Аналог теории

\footnotetext{
^ Работа частично поддержана РФФИ, грант 96-01-006776.
} 
двойственности для групп с инвариантной мерой естественно привел к понятию алгебр в планшерелевой двойственности или планшерелевых троек, обсуждаемых детально в [5] (см. также §2). Именно это понятие и оказывается более адекватным объектам, возникающим в алгебраической комбинаторике. Большое количество примеров таких троек возникает из групп и алгебр.

В настоящей работе предлагается более естественный подход к понятию планшерелевой двойственности. Он базируется на анализе свойств билинейной формы, приводящей две данные полупростые *-алгебры в невырожденную двойственность. Оказывается, что спаривание будет планшерелевым в том и только в том случае, когда оно положительно, однородно и изометрично (см. §2). Свойство изометричности фактически эквивалентно выполнению некоторых соотношений ортогональности в спариваемых алгебрах, в то время как однородность означает, что единица каждой из алгебр определяет одномерное *-представление другой алгебры. Мы планируем определить и изучить неоднородную планшерелеву двойственность (где условие однородности заменяется на более слабое) в отдельной работе. Частный случай такой ситуации возникает при рассмотрении общих клеточных алгебр (не обязательно однородных). Основным результатом работы является следующее утверждение.

ТЕоремА 1. Категории положительных С-алгебр и пар алгебр в планшерелевой двойственности, одна из которьх (например, вторая) коммутативна, эквивалентны.

В этой связи мы заметим, что аксиоматика алгебр в планшерелевой двойственности не требует коммутативности ни одной из алгебр. Имеется много примеров (таких, как квантовые группы), когда обе алгебры некоммутативны. Изучение этого случая представляется привлекательным, поскольку он может рассматриваться как квантовая деформация классической комбинаторики.

Одна из основных задач алгебраической комбинаторики состоит в характеризации $C$-алгебр, возникающих из ассоциативных схем. Условие положительности $C$-алгебры дает необходимое условие существования. На самом деле алгебра смежности ассоциативной схемы есть частный случай однородной клеточной алгебры (см. §3). Мы ставим в соответствие такой алгебре $A \subset \mathrm{Mat}_{n}(\mathbb{C})$ тройку $(A, B,\langle\rangle$,$) , где B$ является *-алгеброй, совпадающей как линейное пространство с $A$ и снабженной покоординатным умножением относительно стандартного базиса в $A$ и комплексным сопряжением в качестве инволющии. Билинейная форма $\langle$,$\rangle определяется формулой \langle a, b\rangle=\frac{1}{n} \operatorname{Tr}\left(a b^{T}\right)$. Доказательство следующего утверждения немедленно вытекает из теоремы 1 и предложения 3.6, приведенного ниже.

ТЕорема 2. Тройка $(A, B,\langle\rangle$,$) является планшерелевой для любой од-$ нородной клеточной алгебры $A$.

Еще одно приложение обсуждаемых объектов связано с многозначными инволютивными группами (см. [2]).

Предварительная версия результатов этой работы изложена в [3]. 


\section{§2. Алгебры в планшерелевой двойственности}

2.1. Спаривание. Мы будем рассматривать тройки $(A, B,\langle\rangle$,$) , где A$, $B$ - полупростые конечномерные $*$-алгебры над $\mathbb{C}$ с единицами $1_{A}$ и $1_{B}$ и

$$
\langle,\rangle: A \times B \rightarrow \mathbb{C}
$$

— билинейная форма, приводящая их в невырожденную двойственность как векторные пространства над $\mathbb{C}$. Мы также предполагаем, что

$$
\left\langle 1_{A}, 1_{B}\right\rangle=1 .
$$

Это предположение не является ограничительным, поскольку легко видеть, что в условиях п. 2.3 мы имеем $\left\langle 1_{A}, 1_{B}\right\rangle \neq 0$ (а в условиях п. 2.2 справедливо даже неравенство $\left.\left\langle 1_{A}, 1_{B}\right\rangle>0\right)$. Под изоморфизмом троек мы понимаем пару изоморфизмов между соответствующими алгебрами, сохраняющих билинейные формы. Элементы алгебр $A$ и $B$ обозначаются через $x, y, z, \ldots$ и $f, g, h, \ldots$ соответственно.

Пусть $\widehat{A}$ и $\widehat{B}$ суть двойственные пространства к $A$ и $B$ соответственно. Тогда спаривание (1), будучи невырожденным, индуцирует линейные изоморфизмы из $\widehat{B}$ в $A$ и из $\widehat{A}$ в $B$. Образ функционала $\chi$ в обоих случаях обозначается через $\widehat{\chi}$.

Используя двойственность между алгебрами $A$ и $B$, можно переносить структуры из $A$ в $B$ и обратно. Например, можно определить коинволюции $\eta_{A}, \eta_{B}$ в $A$ и $B$ как операции, сопряженные к инволюциям в $B$ и $A$ :

$$
\left\langle\eta_{A}(x), f\right\rangle=\overline{\left\langle x, f^{*}\right\rangle}, \quad\left\langle x, \eta_{B}(f)\right\rangle=\overline{\left\langle x^{*}, f\right\rangle}, \quad x \in A, f \in B .
$$

Легко видеть, что $\eta_{A}$ и $\eta_{B}$ являются полулинейными 1-1-отображениями.

Ниже мы определяем алгебры в планшерелевой двойственности (см. [5]). Наш подход, основанный на изучении свойств спаривания, отличается главным образом языком изложения. Соответствие между языками будет обсуждено в п. 2.6.

2.2. Положительное спаривание. Спаривание (1) называется положительным, если конусы

$$
\begin{aligned}
& K_{A}=\left\{x \in A:\langle x, f\rangle \geqslant 0 \quad \forall f \in B^{+}\right\}, \\
& K_{B}=\left\{f \in B:\langle x, f\rangle \geqslant 0 \quad \forall x \in A^{+}\right\}
\end{aligned}
$$

замкнуты относительно умножений и инволюций в алгебрах $A$ и $B$ соответственно. Здесь $A^{+}$(соответственно $B^{+}$) есть конус в $A$ (соответственно $B$ ), натянутый на элементы вида $x x^{*}, x \in A$ (соответственно $f f^{*}, f \in B$ ). В этом случае полулинейные отображения $\eta_{A}$ и $\eta_{B}$, определенные в $(3)$, являются $*$-изоморфизмами алгебр $A$ и $B$ соответственно (см. [5]).

Рассматривая умножения как линейные отображения алгебр

$$
\begin{aligned}
& \Delta_{A}: A \otimes A \rightarrow A, \quad x \otimes y \mapsto x y, \\
& \Delta_{B}: B \otimes B \rightarrow A, \quad f \otimes g \mapsto f g,
\end{aligned}
$$

и переходя к сопряженным отображениям, мы получаем отображения

$$
\nabla_{B}: B \rightarrow B \otimes B, \quad \nabla_{A}: A \rightarrow A \otimes A,
$$


называемые коумножениями. В нашем случае (положительного спаривания) $\nabla_{A}$ и $\nabla_{B}$ являются положительными в том смысле, что

$$
\nabla_{A} A^{+} \subset A^{+} \otimes A^{+}, \quad \nabla_{B} B^{+} \subset B^{+} \otimes B^{+} .
$$

В теории алгебр Хопфа и биалгебр одно из коумножений (а тогда автоматически и второе) есть по определению *-гомоморфизм алгебр (см. [6]). Поскольку *-гомоморфизм является положительным отображением, аксиоматика положительных троек представляет собой естественное ослабление аксиоматики алгебр Хопфа. Эта тема будет более детально изучена в следующей статье.

2.3. Однородное спаривание. Спаривание (1) называется однородныл, если множества

$$
\begin{aligned}
& I_{A}=\left\{x \in A:\left\langle x, 1_{B}\right\rangle=0\right\}, \\
& I_{B}=\left\{f \in B:\left\langle 1_{A}, f\right\rangle=0\right\}
\end{aligned}
$$

являются двусторонними идеалами в алгебрах $A$ и $B$ соответственно. В этом случае (см. (2)) отображения $x \mapsto\left\langle x, 1_{B}\right\rangle$ и $f \mapsto\left\langle 1_{A}, f\right\rangle$ суть $*$-гомоморфизмы алгебр $A$ и $B$ в $\mathbb{C}$. Будучи двусторонними идеалами коразмерности один, $I_{A}$ и $I_{B}$ определяют примитивные центральные идемпотенты $m_{A} \in A$ и $m_{B} \in B$, для которых

$$
\begin{array}{ll}
x m_{A}=m_{A} x=\left\langle x, 1_{B}\right\rangle m_{A}, & x \in A, \\
f m_{B}=m_{B} f=\left\langle 1_{A}, f\right\rangle m_{B}, & f \in B,
\end{array}
$$

и, в частности, $\left\langle m_{A}, 1_{B}\right\rangle=\left\langle 1_{A}, m_{B}\right\rangle=1$. В терминах отображений $\nabla_{A}$ и $\nabla_{B}$ однородность эквивалентна равенствам

$$
\nabla_{A}\left(1_{A}\right)=1_{A} \otimes 1_{A}, \quad \nabla_{B}\left(1_{B}\right)=1_{B} \otimes 1_{B} .
$$

Формулы (4) и (5) показывают, что элементы $m_{A}$ и $m_{B}$ могут рассматриваться в качестве аналогов нормализованных инвариантных мер в $A$ и $B$ соответственно. Например, пусть $A=\mathbb{C}[G]-$ групповая алгебра конечной группы $G$ и $B=C(G)$ - алгебра комплексных функций на $G$ с поточечным умножением. Определим двойственность по формуле

$$
\langle x, f\rangle=\sum_{g \in G} x(g) f(g), \quad x \in A, f \in B .
$$

В этом случае

$$
m_{A}=\frac{1}{\# G} \sum_{g \in G} g, \quad m_{B}=\frac{1}{\# G} \sum_{\pi \in G^{\wedge}}(\operatorname{dim} \pi) \chi_{\pi}
$$

являются мерой Хаара на $G$ и мерой Планшереля на $G^{\wedge}$ соответственно, где $\chi_{\pi}$ - характер неприводимого комплексного представления $\pi$ группы $G$ и $\operatorname{dim} \pi$ - его размерность.

2.4. Следы. Линейный функционал $\chi: A \rightarrow \mathbb{C}$ называется следом (feasible trace в [4]) на $A$, если $\chi(x y)=\chi(y x)$ для всех $x, y \in A$. В этом случае

$$
\chi=\sum_{\pi} m(\pi, \chi) \chi_{\pi}, \quad \chi_{\pi}(x)=\operatorname{Tr}(\pi(x)),
$$


где $m(\pi, \chi) \in \mathbb{C}$, а $\pi$ пробегает полную систему попарно неэквивалентных неприводимых представлений алгебры $A$. Такой функционал $\chi$ определяет полуторалинейную форму

$$
(x, y)_{\chi}=\chi\left(x y^{*}\right), \quad x, y \in A,
$$

так что $(u x v, u y v)_{\chi}=(x, y)$ для всех $u, v \in A$ с $u u^{*}=v v^{*}=1_{A}$. Обратно, всякая полуторалинейная форма с этим свойством совпадает с $(,)_{\chi}$ для единственным образом определенного следа $\chi$. Отметим, что форма (7) сопряженносимметрична тогда и только тогда, когда $m(\pi, \chi)$ является вещественным числом для всех $\pi$ (эквивалентно, $\chi\left(x^{*}\right)=\overline{\chi(x)}$ для всех $x$ ), и положительно определена тогда и только тогда, когда $m(\pi, \chi)>0$ для всех $\pi$ (эквивалентно, $\chi\left(x x^{*}\right)>0$ для всех $\left.x \neq 0\right)$. В последнем случае мы говорим, что $\chi$ является положительным.

Пусть теперь $\chi_{A}$ и $\chi_{B}$ - положительные следы на алгебрах $A$ и $B$, спаренных посредством (1). Тогда $A$ и $B$ могут рассматриваться как гильбертовы пространства со скалярными произведениями $(,)_{A}=(,)_{\chi_{A}}$ и $(,)_{B}=$ $(,)_{\chi_{B}}$. Поскольку спаривание $(1)$ невырождено, имеются преобразования Фурье $F_{A}: A \rightarrow B$ и $F_{B}: B \rightarrow A$, определенные формулами

$$
\begin{array}{ll}
\left\langle y, F_{A}(x)\right\rangle=\left(x, y^{*}\right)_{A}, & y \in A, \\
\left\langle F_{B}(f), g\right\rangle=\left(f, g^{*}\right)_{B}, & g \in B .
\end{array}
$$

Из определения следует, что

$$
F_{A}\left(1_{A}\right)=\widehat{\chi}_{A}, \quad F_{B}\left(1_{B}\right)=\widehat{\chi}_{B},
$$

где элементы $\widehat{\chi}_{A} \in B$ и $\widehat{\chi}_{B} \in A$ отвечают $\chi_{A}$ и $\chi_{B}$ при отождествлении $\widehat{A}$ с $B$ и $\widehat{B}$ с $A$ (см. п. 2.1).

2.5. Изометричное спаривание. Будем говорить, что спаривание (1) является изометричнылм относительно положительных следов $\chi_{A}$ и $\chi_{B}$, если отображения $F_{A}$ и $F_{B}$, определенные выше, кратны изометриям между гильбертовыми пространствами $\left(A,(,)_{A}\right)$ и $\left(B,(,)_{B}\right)$. Спаривание называется изометричным, если оно изометрично относительно некоторых положительных следов.

Прямое вычисление показывает, что изометричность относительно $\chi_{A}$ и $\chi_{B}$ влечет за собой равенство

$$
F_{B} F_{A}=M\left(\eta_{A}^{*}\right)^{-1}, \quad F_{A} F_{B}=M\left(\eta_{B}^{*}\right)^{-1},
$$

где $\eta_{A}^{*}(x)=\eta_{A}(x)^{*}, \eta_{B}^{*}(f)=\eta_{B}(f)^{*}$ (см. $\left.(3)\right)$ и

$$
M=\left\|F_{A}\right\|^{2}=\left\|F_{B}\right\|^{2}=\left\langle\widehat{\chi}_{B} \eta_{A}^{*}\left(1_{A}\right), \widehat{\chi}_{A}\right\rangle=\left\langle\widehat{\chi}_{B}, \widehat{\chi}_{A} \eta_{B}^{*}\left(1_{B}\right)\right\rangle
$$

( $\|$ || обозначает операторную норму). Кроме того, из (9) и (8) вытекает, что $\eta_{A}$ и $\eta_{B}$ являются изометриями, для которых выполняются равенства

$$
F_{A}\left(\widehat{\chi}_{B}\right)=M \eta_{B}\left(1_{B}\right), \quad F_{B}\left(\widehat{\chi}_{A}\right)=M \eta_{A}\left(1_{A}\right) .
$$


Пусть $\pi: B \rightarrow \operatorname{Mat}_{n_{\pi}}(\mathbb{C})$ - неприводимое $*$-представление алгебры $B$. Обозначим через $f_{i j}^{\pi}$ элемент из $B$, соответствующий матричной единице $e_{i j}$ алгебры $\operatorname{Mat}_{n_{\pi}}(\mathbb{C}), i, j=1, \ldots, n_{\pi}$. Линейный базис $\left\{f_{i j}^{\pi}\right\}_{i, j, \pi}$ алгебры $B$ определяет двойственный базис в $\widehat{B}$ и потому линейный базис $\left\{x_{i j}^{\pi}\right\}_{i, j, \pi}$ в $A$. Из определений п. 2.4 получаем, что

$$
\left(f_{i, j}^{\pi}, f_{k, l}^{\rho}\right)_{B}=\delta_{\pi, \rho} m\left(\pi, \chi_{B}\right) \operatorname{Tr}\left(f_{i, j}^{\pi} f_{k, l}^{\rho}\right)=\delta_{\pi, \rho} \delta_{i, k} \delta_{j, l} m\left(\pi, \chi_{B}\right)
$$

и

$$
F_{B}\left(f_{i, j}^{\pi}\right)=m\left(\pi, \chi_{B}\right) x_{j, i}^{\pi} .
$$

Таким образом, формула $\left(F_{B}(f), F_{B}(g)\right)_{A}=\left\|F_{B}\right\|^{2}(f, g)_{B}, f, g \in B$, эквивалентна следующим соотношениям ортогональности в алгебре $A$ (см. (10)):

$$
\left(x_{i j}^{\pi}, x_{k l}^{\rho}\right)_{A}=\delta_{\pi, \rho} \delta_{i, k} \delta_{j, l} \frac{M}{m\left(\pi, \chi_{B}\right)} .
$$

Аналогичные соотношения ортогональности легко выписываются также для алгебры $B$.

2.6. Планшерелева двойственность. Соберем вместе свойства спаривания (1), описанные в пп. 2.2, 2.3 и 2.5 .

ОПРЕДЕлЕНИЕ 2.1. Будем говорить, что две полупростые $*$-алгебры $A$ и $B$, спаренные посредством (1), находятся в (однородной) планшерелевой двойственности, если спаривание положительно, однородно и изометрично. В этом случае спаривание $\langle$,$\rangle и тройка (A, B,\langle\rangle$,$) называются планшерелевьли.$

Рассмотрим планшерелеву тройку $(A, B,\langle\rangle$,$) . Тогда найдутся положитель-$ ные следы $\chi_{A}$ и $\chi_{B}$, относительно которых спаривание является изометричным. Не умаляя общности, будем считать, что

$$
\chi_{A}\left(1_{A}\right)=\chi_{B}\left(1_{B}\right)=1 .
$$

Следующее утверждение описывает соотношение между основными инвариантами тройки и фактически устанавливает единственность таких $\chi_{A}$ и $\chi_{B}$.

ЛЕмма 2.2. Имеют место такие утверждения:

(1) $m_{A}=\widehat{\chi}_{B}, m_{B}=\widehat{\chi}_{A}$;

(2) $\left\|F_{A}\right\|^{2}=\left\|F_{B}\right\|^{2}=\left\langle m_{A}, m_{B}\right\rangle$, в частности, $\left\langle m_{A}, m_{B}\right\rangle>0$;

(3) $F_{A}\left(1_{A}\right)=m_{B}, F_{B}\left(1_{B}\right)=m_{A}$, а также $F_{A}\left(m_{A}\right)=M 1_{B}, F_{B}\left(m_{B}\right)=$ $M 1_{A}$, где $M=\left\langle m_{A}, m_{B}\right\rangle$.

ДокаЗАТЕЛЬСТВо. Поскольку $\left\langle m_{A}, 1_{B}\right\rangle=1=\chi_{B}\left(1_{B}\right)$ (см. П. 2.3 и (12)), мы заключаем, что $m\left(\chi_{B}\right)=m_{A}+x$ для некоторого $x \in I_{A}$. По $(9)$ с учетом того, что $\eta_{B}\left(1_{B}\right)=1_{B}$ (см. п. 2.2$)$, мы имеем

$$
M\left\langle x^{*}, 1_{B}\right\rangle=\left\langle x^{*} F_{A} F_{B}\left(\eta_{B}^{*}\left(1_{B}\right)\right)\right\rangle=\left\langle x^{*} F_{A} F_{B}\left(1_{B}\right)\right\rangle .
$$

Кроме того, согласно (8) и определениям $F_{A}$ и $m_{A}$,

$$
\left\langle x^{*}, F_{A} F_{B}\left(1_{B}\right)\right\rangle=\left\langle x^{*}, F_{A}\left(\widehat{\chi}_{B}\right)\right\rangle=\left(\widehat{\chi}_{B}, x\right)_{A}=\left(m_{A}+x, x\right)_{A}=(x, x)_{A} .
$$

Из включения $x^{*} \in I_{A}$ следует, что $(x, x)_{A}=0$, откуда $x=0$. Таким образом, $\widehat{\chi}_{B}=m_{A}$. Аналогично, $\widehat{\chi}_{A}=m_{B}$, что доказывает первое утверждение. Это 
вместе с (10) влечет за собой второе утверждение. Наконец, последнее утверждение следует из первого и равенств (8), (11).

Если алгебры $A$ и $B$ находятся в планшерелевой двойственности, то естественное отождествление пространств $A, B, \widehat{A}, \widehat{B}$ приводит к гильбертову пространству с двумя структурами *-алгебры. Более того, соответствующее скалярное произведение инвариантно относительно левого и правого действия обеих унитарных групп, индуцированных этими структурами. Единица относительно одной из этих структур является кратным примитивного центрального идемпотента относительно второй структуры. Блок-симплекс состояний (см. [7]) (в частном случае - симплекс характеров) в одной из этих алгебр замкнут относительно умножения и инволюции во второй алгебре. Такая схема дает возможность устанавливать связи с алгебраической комбинаторикой, которые будут рассмотрены в $\S 3$.

2.7. Связь с работами $[5,7]$. В этом пункте мы сравниваем понятия, рассмотренные выше, с понятиями алгебр в положительной и планшерелевой двойственности, введенными и изученными в $[7,5]$.

Алгебры $A$ и $B$ находятся в (однородной) положительной двойственности относительно невырожденного спаривания $\langle\rangle,((A, B,\langle\rangle$,$) есть (однородная)$ положительная тройка), если множества

$$
\begin{aligned}
& P_{A}=\left\{x \in A:\left\langle x, 1_{B}\right\rangle=1,\langle x, f\rangle \geqslant 0 \quad \forall f \in B^{+}\right\}, \\
& P_{B}=\left\{f \in B:\left\langle 1_{A}, f\right\rangle=1,\langle x, f\rangle \geqslant 0 \quad \forall x \in A^{+}\right\}
\end{aligned}
$$

замкнуты относительно умножений и инволюций в алгебрах $A$ и $B$ соответственно. Легко видеть, что такая ситуация возникает, если спаривание положительно и однородно. Обратно, если $(A, B,\langle\rangle$,$) - положительная тройка, то$ спаривание $\langle$,$\rangle , очевидно, положительно. Более того, если x, y \in I_{A}$, причем $1_{A}+x, 1_{A}+y \in A^{+}$, то

$$
\left(1_{A}+x\right)\left(1_{A}+y\right)=1_{A}+x+y+x y \in P_{A} .
$$

Это показывает, что $x y,\left(1_{A}+x\right) y, x\left(1_{A}+y\right) \in I_{A}$, и, следовательно, $I_{A}$ является двусторонним идеалом в $A$. Аналогично, $I_{B}$ есть двусторонний идеал в $B$, т. е. спаривание однородно.

Пусть теперь $(A, B,\langle\rangle$,$) - положительная тройка. Согласно (4) и (5),$ элементы $m_{A}$ и $m_{B}$ являются нулями полугрупп $P_{A}$ и $P_{B}$. В статье [5] в предположении, что они центральны и невырожденны, было определено понятие алгебр в планшерелевой двойственности. Оно с точностью до языка совпадает с понятием, введенным в п. 2.6. Действительно, тот факт, что каждый нуль является центральным, означает, что

$$
\begin{array}{ll}
\left\langle m_{A}, f g\right\rangle=\left\langle m_{A}, g f\right\rangle, & f, g \in B, \\
\left\langle x y, m_{B}\right\rangle=\left\langle y x, m_{B}\right\rangle, & x, y \in A .
\end{array}
$$

В этом случае имеют место разложения

$$
m_{A}=\sum_{\pi} m_{A}(\pi) \hat{\chi}_{\pi}, \quad m_{B}=\sum_{\pi} m_{B}(\pi) \widehat{\chi}_{\pi},
$$


где $\pi$ пробегает полные системы попарно неэквивалентных неприводимых представлений алгебр $A$ и $B$ соответственно (см. (6) и п. 2.1). Это, в свою очередь, означает, что $m_{A}=\widehat{\chi}_{B}, m_{B}=\widehat{\chi}_{A}$ для некоторых следов на $B$ и $A$, удовлетворяющих (12). В то же время $m_{A}(\pi)$ и $m_{B}(\pi)$ совпадают с коэффициентами $m\left(\pi, \chi_{B}\right)$ и $m\left(\pi, \chi_{A}\right)$ из $(13)$. Кроме того, невырожденность нулей означает, что $m_{A}(\pi)$ и $m_{B}(\pi)$ в $(13)$ являются положительными вещественными числами. (Как отмечено в [5], невырожденность любого из нулей $m_{A}$ и $m_{B}$ влечет за собой невырожденность второго и эквивалентна неравенству $\left\langle m_{A}, m_{B}\right\rangle>0$.) Это эквивалентно тому, что следы $\chi_{A}$ и $\chi_{B}$ являются положительными. Наконец, свойство изометричности относительно $\chi_{A}$ и $\chi_{B}$ для положительной тройки $(A, B,\langle\rangle$,$) выражает в точности тот факт, что A$ и $B$ находятся в планшерелевой двойственности в смысле [5].

Следующее простое утверждение, доказанное в [5], будет использовано в $\S 4$.

ПРЕДЛОЖЕНИЕ 2.3. Пусть $(A, B,\langle\rangle$,$) - положительная тройка с не-$ вырожденными центральными нулями и коммутативной алгеброй В. Следующие условия эквивалентны:

(1) тройка $(A, B,\langle\rangle$,$) планшерелева;$

(2) выполняются соотношения ортогональности для алгебрьл $A$ :

$$
\left(x_{p}, x_{q}\right)_{A}=\frac{M}{m_{A}(p)} \delta_{p, q},
$$

где $p, q$ - примитивнье иентральнье идемпотентьл алгебры $B, x_{p}=\widehat{\chi}_{\pi}$, $x_{q}=\widehat{\chi}_{\rho}, m_{A}(p)=m_{A}(\pi)(\pi, \rho$ - неприводимьее представления алгебрь $B$, отвечающие $p$ u q) и $M=\left\langle m_{A}, m_{B}\right\rangle$.

ДокАЗАТЕЛЬСТво. Из условия следует, что $m_{A}$ и $m_{B}$ определяют по двойственности некоторые положительные следы $\chi_{B}$ и $\chi_{A}$, удовлетворяющие $(12)$. Поэтому равенство (14) эквивалентно тому, что отображение $F_{B}$ изометрично (см. п. 2.5). Поскольку тройка положительна, инволютивное отображение $\eta_{A}$ является изоморфизмом алгебры $A$ (см. п. 2.2) и потому изометрией гильбертова пространства $\left(A,(,)_{A}\right)$. Таким образом, по $(9)$ отображение $F_{A}$ также изометрично и, следовательно, тройка $(A, B,\langle\rangle$,$) планшерелева.$

\section{§3. Положительные $C$-алгебры}

Пусть $A$ - ассоциативная алгебра над $\mathbb{C}$ с единицей 1 , полулинейным инволютивным антиавтоморфизмом * и выделенным линейным базисом $\mathscr{R}$. Структурные константы этой алгебры в базисе $\mathscr{R}$ определяются формулой $r \cdot s=$ $\sum_{t \in \mathscr{R}} c_{r, s}^{t} \cdot t$ для $r, s \in \mathscr{R}$.

ОПРЕДЕлЕниЕ 3.1. Пара $(A, \mathscr{R})$ называется $C$-аләеброй ${ }^{1}$, если выполнены следующие условия:

(С1) $1 \in \mathscr{R}$ и $\mathscr{R}^{*}=\mathscr{R}$;

(C2) структурные константы $c_{r, s}^{t}$ алгебры $A$ относительно базиса $\mathscr{R}$ вещественны;

${ }^{1} \mathrm{~K}$ сожалению, термин «C-алгебра» представляется крайне неудачным, так как есть риск смешения его с терминами $C^{*}$-алгебра и $\mathbb{C}$-алгебра. 
(С3) для всех $r, s \in \mathscr{R}$ имеет место равенство $c_{r, s}^{1}=d(r) \delta_{r^{*}, s}$, где $d(r)>0$ и $\delta$ - символ Кронекера;

(С4) линейный функционал на $A$, определяемый отображением $r \mapsto d(r)$, $r \in \mathscr{R}$, является одномерным *-представлением алгебры $A$; в частности, $d(r)=$ $d\left(r^{*}\right)$ для всех $r \in \mathscr{R}$.

ЗАмЕчАниЕ 3.2. В алгебраической комбинаторике (см. [1]) понятие $C$-алгебры используется в основном для коммутативных алгебр.

Под изоморфизмом $C$-алгебр мы будем понимать $*$-изоморфизм соответствующих алгебр, сохраняющий базисы.

Определим в $A$ покоординатное умножение о относительно базиса $\mathscr{R}$, полагая

$$
r \circ s=\delta_{r, s} r, \quad r, s \in \mathscr{R} .
$$

Очевидно, что относительно этого умножения $A$ является коммутативной ассоциативной алгеброй над $\mathbb{C}$ с единицей $J=\sum_{r \in \mathscr{R}} r$.

ОПРЕДЕЛЕНИЕ 3.3 . Будем называть $C$-алгебру $\mathscr{A}=(A, \mathscr{R})$ положительной, если

(P1) все структурные константы $c_{r, s}^{t}$ алгебры $A$ относительно базиса $\mathscr{R}$ неотрицательны;

(P2) конус $A^{+}=\left\{a a^{*}: a \in A\right\}$ неотрицательных элементов алгебры $A$ замкнут относительно умножения $\circ$.

ЗАмЕчАниЕ 3.4. Следующий пример показывает, что условие (P2) не вытекает из (P1). В коммутативной $C$-алгебре $(A,\{1, r, s\})$ с умножением

$$
r^{2}=m \cdot 1+(m-1) \cdot s, \quad s^{2}=m \cdot 1+r+(m-2) \cdot s, \quad r s=s r=(m-1) \cdot r+s
$$

и инволюцией $*$, такой, что $r^{*}=r, s^{*}=s$, условие (P1), очевидно, выполнено для всех $m \geqslant 2$. С другой стороны, условие (P2) нарушается для каждого $m>2$. (Такие примеры были, по-видимому, известны и ранее.)

Действительно, прямым вычислением нетрудно показать, что коэффициент при $p$ разложения элемента $p \circ p$ по базису из примитивных идемпотентов алгебры $A$ строго меньше нуля для одного из ее примитивных идемпотентов $p$. Отсюда следует, что $p \circ p \notin A^{+}$(см. предложение 3.5$)$.

Условие (Р1) фактически означает замкнутость конуса неотрицательных элементов по отношению к базису $\mathscr{R}$ (т. е. октанта) относительно умножения в $A$. Условие (P2) есть в точности дуальное условие. В использовании этой симметрии и состояла идея определения положительной двойственности в [7].

Проверка условия (P1) тривиальна, в то время как проверка (P2) для случая некоммутативной алгебры $A$ представляет непростую задачу. Следует отметить, что для коммутативных $C$-алгебр это условие легко переписывается в терминах констант Крейна. Напомним, что константы Крейна коммутативной $C$-алгебры $\mathscr{A}=(A, \mathscr{R})$ суть ее двойственные структурные константы. Более точно, константы Крейна $c_{p_{1}, p_{2}}^{p}, p_{1}, p_{2}, p \in \operatorname{Spec}(A)$, определяются формулой

$$
p_{1} \circ p_{2}=\frac{1}{n} \sum_{p \in \operatorname{Spec}(A)} c_{p_{1}, p_{2}}^{p} p,
$$


где $n=\sum_{r \in \mathscr{R}} d(r)$, а $\operatorname{Spec}(A)$ - множество примитивных центральных идемпотентов алгебры $A$.

ПРЕДЛОЖЕНИЕ 3.5. Положительность коммутативной C-алгебры равносильна неотрицательности ее структурных констант и констант Крейна.

ДокАЗАтЕльство. Пусть $\mathscr{A}=(A, \mathscr{R})$ - коммутативная $C$-алгебра. Тогда легко видеть, что

$$
A^{+}=\left\{\sum_{p \in \operatorname{Spec}(A)} \alpha_{p} p: \alpha_{p} \geqslant 0\right\} .
$$

Однако по определению констант Крейна их неотрицательность в точности означает, что конус $A^{+}$замкнут относительно умножения $\circ$.

Для $C$-алгебр, возникаюших из коммутативных схем отношений, константы Крейна неотрицательны (условие Крейна), что дает необходимое условие существования таких схем. Условие (Р2) фактически обобщает условие Крейна на некоммутативный случай (см. предложение 3.6 ниже). Примеры таких алгебр возникают из групповых рассмотрений: таковыми являются групповая алгебра и некоторые ее подалгебры (см. $[1,7,5])$.

Содержательные примеры положительных $C$-алгебр поставляют клеточные (когерентные) алгебрыл, введенные в [8] (и независимо в [4]) как подалгебры в $\operatorname{Mat}_{n}(\mathbb{C})$, замкнутые относительно адамарова (покомпонентного) умножения и эрмитова сопряжения и содержащие матрицу $J_{n}$, в которой во всех позициях стоят 1. Такими алгебрами являются, в частности, централизаторные алгебры групп перестановок и алгебры смежности когерентных конфигураций. В произвольной клеточной алгебре $A \subset \operatorname{Mat}_{n}(\mathbb{C})$ имеется однозначно определенный линейный базис $\mathscr{R}(A)$, состоящий из $\{0,1\}$-матриц, в сумме дающих $J_{n}$, и замкнутый относительно транспонирования. Клеточная алгебра $A$ называется однородной, если ее стандартный базис $\mathscr{R}(A)$ содержит единичную матрицу. Примерами однородных клеточных алгебр являются централизаторные алгебры транзитивных групп перестановок и алгебры Боуза-Меснера схем отношений (необязательно коммутативных).

Если $A$ - однородная клеточная алгебра, то пара $(A, \mathscr{R}(A))$, как легко видеть, является $C$-алгеброй (возможно, некоммутативной), у которой все числа $d(r), r \in \mathscr{R}(A)$, являются целыми и положительными. С другой стороны, такая $C$-алгебра $(A, \mathscr{R})$ вместе с точным *-представлением

$$
A \rightarrow \operatorname{Mat}_{n}(\mathbb{C}), \quad n=\sum_{r \in \mathscr{R}} d(r),
$$

при котором умножение ○ в $A$ (см. (15)) переходит в адамарово умножение матриц, может рассматриваться как однородная клеточная алгебра.

ПрЕДЛОЖЕНИЕ 3.6. Если $A$ - однородная клеточная алгебра, то С-алгебра $(A, \mathscr{R}(A))$ является положительной.

ДоказАтельство. Ясно, что структурные константы $C$-алгебры $(A, \mathscr{R}(A))$ суть неотрицательные целые числа. С другой стороны, легко видеть, что конус $A^{+}$в этом случае состоит из неотрицательно определенных эрмитовых матриц, содержащихся в $A$. Поэтому условие (Р2) вытекает из хорошо известного 
факта, что адамарово произведение неотрицательно определенных эрмитовых матриц является неотрицательно определенной эрмитовой матрицей (см., например, лемму 3.9 в [1]).

\section{§4. Доказательство теоремы 1}

4.1. Планшерелевость положительных $C$-алгебр. Пусть $\mathscr{A}=(A, \mathscr{R})$ - положительная $C$-алгебра. Введем в линейном пространстве $B=A$ структуру коммутативной *-алгебры с умножением о (см. (15)) и сопряжением $*$, $r^{*}=r, r \in \mathscr{R}$. При этом, как легко видеть, $\operatorname{Spec}(B)=\mathscr{R}$. Определим спаривание между $A$ и $B$, полагая

$$
\langle r, s\rangle_{\mathscr{R}}=\langle r, s\rangle=\delta_{r, s} d(r), \quad r, s \in \mathscr{R} .
$$

Покажем, что тройка $\mathscr{T}(\mathscr{A})=(A, B,\langle\rangle$,$) является планшерелевой.$

Легко видеть, что

$$
P_{A}=\left\{x=\sum_{r \in \mathscr{R}} \alpha_{r} r: \alpha_{r} \geqslant 0, d(x)=1\right\} .
$$

Поэтому замкнутость множества $P_{A}$ относительно умножения и сопряжения в алгебре $A$ следует из неотрицательности ее структурных констант относительно $\mathscr{R}$ (условие $(\mathrm{P} 1))$ и условия $(\mathrm{C} 4)$. С другой стороны,

$$
P_{B}=\left\{x \in A^{+}: \alpha_{1}(x)=1\right\},
$$

где $\alpha_{1}(x)$ - коэффициент при $1_{A}$ в разложении $x$ по $\mathscr{R}$. Таким образом, замкнутость множества $P_{B}$ относительно умножения и сопряжения в алгебре $B$ следует из условия (Р2). Тем самым доказано, что $\mathscr{T}(\mathscr{A})$ является положительной тройкой.

Рассмотрим нули $m_{A}$ и $m_{B}$ алгебр $A$ и $B$. Нетрудно проверить, что

$$
m_{A}=\frac{1}{n} \sum_{r \in \mathscr{R}} r, \quad m_{B}=1_{A},
$$

где $n=\sum_{r \in \mathscr{R}} d(r)=1 / M$. Центральность $m_{A}$ очевидна в силу коммутативности алгебры $B$, в то время как центральность $m_{B}$ следует из условий $(\mathrm{C} 3),(\mathrm{C} 4)$. Из тех же условий вытекает, что

$$
m_{A}(r)=\left\langle m_{A}, r\right\rangle=d(r) / n>0, \quad r \in \operatorname{Spec}(B)=\mathscr{R} .
$$

Это доказывает невырожденность $m_{A}$. Для завершения доказательства планшерелевости в силу предложения 2.3 достаточно проверить соотношения ортогональности (14). В нашем случае они принимают вид

$$
(r / d(r), s / d(s))_{A}=\delta_{r, s} / d(r),
$$

что совпадает с (C3) по определению $(,)_{A}$.

4.2. Положительность $\boldsymbol{C}$-алгебры, соответствующей планшерелевой тройке. Обратно, пусть $\mathscr{T}=(A, B,\langle\rangle$,$) - планшерелева тройка,$ причем алгебра $B$ коммутативна. Положим

$$
\mathscr{R}=\left\{r(p) \in A: r(p)=\left(m_{A}(p) / M\right) x_{p}, p \in \operatorname{Spec}(B)\right\},
$$


где $m_{A}(p), M$ и $x_{p}$ определены в предложении 2.3. Докажем, что пара $\mathscr{A}(\mathscr{T})=$ $(A, \mathscr{R})$ является положительной $C$-алгеброй. Отметим, что $\mathscr{R}-$ линейный базис алгебры $A$ в силу невырожденности $m_{A}$. Ниже мы полагаем $d(r)=$ $m_{A}(p) / M$ для $r=r(p)$.

Так как $1_{A}=r\left(m_{B}\right)$ (см. утверждение (3) леммы 2.2$)$, то $1_{A} \in \mathscr{R}$. Замкнутость $\mathscr{R}$ относительно * следует из того факта, что отображение $\eta_{B}$, определенное в (3), является *-изоморфизмом алгебры $B$ (см. [5]). Это доказывает (C1). Далее, из соотношений ортогональности (14) следует, что

$$
(r, s)_{A}=d(r) \delta_{r, s}, \quad r, s \in \mathscr{R} .
$$

По определению скалярного произведения $(,)_{A}$ имеем

$$
c_{r, s}^{1}=\left\langle r s, m_{B}\right\rangle=\left(r, s^{*}\right)_{A}=d(r) \delta_{r, s^{*}},
$$

откуда следует (С3), поскольку $d(r)>0$ в силу невырожденности $m_{A}$. Последняя формула показывает, что

$$
c_{r, s}^{t}=\left\langle r s t^{*}, m_{B}\right\rangle / d(t), \quad r, s, t \in \mathscr{R} .
$$

Заметим, что в силу коммутативности $B$

$$
B^{+}=\left\{\sum_{p \in \operatorname{Spec}(B)} \alpha_{p} p: \alpha_{p} \geqslant 0\right\} .
$$

Отсюда по определению $\mathscr{R}$ заключаем, что $\mathscr{R} \subset K_{A}$. Таким образом, условие $(\mathrm{C} 2)$ и, более того, неотрицательность $c_{r, s}^{t}$, следуют из замкнутости конуса $K_{A}$ относительно умножения и сопряжения и того факта, что $m_{B} \in B^{+}$. Свойство (C4) вытекает из формулы

$$
d(r)=\langle r, p\rangle=\left\langle r, 1_{B}\right\rangle
$$

где $r=r(p), p \in \operatorname{Spec}(B)$, и того, что отображение $x \mapsto\left\langle x, 1_{B}\right\rangle$ является *-характером алгебры $A$ (см. п. 2.3).

Для доказательства положительности $C$-алгебры $\mathscr{A}(\mathscr{T})$ заметим, что условие (P1) было фактически проверено при доказательстве условия (C2). С другой стороны, легко видеть, что отображение $\operatorname{Spec}(B) \rightarrow \mathscr{R}, p \mapsto r(p)$, продолжается до линейного изоморфизма алгебры $B$ на $A$, переводящего умножение в $B$ в умножение о в $A$, при котором $K_{B}$ переходит в $A^{+}$. Таким образом, условие (Р2) следует из замкнутости конуса $K_{B}$ относительно умножения в $B$.

4.3. Эквивалентность категорий. Проверим, что отображения $\mathscr{T} \rightarrow$ $\mathscr{A}(\mathscr{T})$ и $\mathscr{A} \rightarrow \mathscr{T}(\mathscr{A})$ задают эквивалентность соответствующих категорий. Ясно, что $\mathscr{A}(\mathscr{T}(\mathscr{A}))=\mathscr{A}$. С другой стороны, пусть $\mathscr{T}=(A, B,\langle\rangle)-$, планшерелева тройка с коммутативной алгеброй $B$. Тогда в планшерелевой тройке $\mathscr{T}(\mathscr{A}(\mathscr{T}))$ первая компонента совпадает с алгеброй $A$, в то время как вторая компонента представляет собой линейное пространство $A$ с коммутативным умножением о (см. (15)). Изоморфизм между этими тройками задается тождественным отображением алгебры $A$ и линейным изоморфизмом $B \rightarrow A$, определенным в конце п. 4.2. Теорема доказана. 


\section{ЛитеРАТУРА}

1. Баннаи Э., Ито Т. Алгебраическая комбинаторика 1. Схемы отношений. Мир, M., 1987.

2. Бухитабер В. М., Вериик А. М., Евдокимов С. А., Пономаренко И. Н. Комбинаторные алгебры и многозначные инволютивные группы, Функц. анализ и его прил., 30, вып. 3, 12-18 (1996).

3. Вериик A. М., Евдокимов С. А., Пономаренко И. Н. С-алгебры и алгебры в планшерелевой двойственности. Зап. научн. сем. ПОМИ, 240, 53-66 (1997).

4. Higman D. G. Coherent algebras. Linear Algebra Appl., 93, 209-239, (1987).

5. Керов С. В. Двойственность конечномерных *-алгебр. Вестн. ЛГУ, сер. матем., 7, 23-29, (1974).

6. Kassel Ch. Quantum Groups, Springer-Verlag, 1994.

7. Вершик A. М. Геометрическая теория состояний, граница фон Неймана, двойственность $C^{*}$-алгебр. Зап. научн. семин. ПОМИ, 29, 147-154, (1972).

8. Weisfeiler B. Ju. (ed.) On construction and identification of graphs. Springer Lecture Notes, Vol. 558, 1976.

С.-Петербургское отделение Математического института им. В. А. Стеклова РАН

Поступило в редакцию 5 сентября 1997 г.

С.-Петербургский институт

информатики и автоматизации РАН

С.-Петербургское отделение Математического

института им. В. А. Стеклова РАН 